DRAL is part of the Engineering and Physical Sciences Research Council

The Engineering and Physical Sciences Research Council

does not accept any responsibility for loss or damage arising from the use of information contained in any of its reports or in any communication about its tests or investigations 


\title{
Non-linear viscoelastic behaviour
}

Irving $\mathrm{A} \mathrm{D}^{+}$, Watson $\mathrm{S} \mathrm{J}^{+}$, Speet $\mathrm{L} \mathrm{J} \mathrm{Jo}$, Cunliffe $\mathrm{N}^{+}$and Dewson $\mathrm{T}^{*}$

+Nonlinear Time Series Group, Energy Research Unit, Rutherford Appleton Laboratory, Chilton, Didcot, Oxon, OX11 0QX, UK

oDepartment of Civil Engineering, Delft Technical University, Delft, The Netherlands.

*Department of Mathematics, University Walk, University of Bristol, Bristol, BS8 1TW, UK.

\begin{abstract}
This paper is concerned with the development of a quantitative analysis method that can extract the mixed linear and non-linear dynamical response of a viscoelastic process in composite materials. A tractable set of simultaneous equations with well behaved coefficients can be generated by taking time series moments of a suitably truncated Volterra series expansion. This moment hierarchy is a set of inhomogeneous non-linear integral equations, based on a vector multidimensional convolution form of the Volterra functional series expansion. The hierarchy developed is used to analyse the time dependent viscoelastic properties of fibre reinforced composite materials. Estimates of the temporal response of the measured stress to the measured mechanical force were then used to predict the out of sample stress field values. It is demonstrated that the response functions provided a good representation of the viscoelastic process. Generally speaking, the response functions estimated from the data are used to determine the dynamic and steady state transport coefficients, which can, be used to develop either an empirical field theory of the phenomena or alternatively be used in the design process.
\end{abstract}




\section{Introduction}

In ideal elastic bodies any deformation below the yield point is assumed to be reversible. In real materials the motion induced by the applied mechanical force is irreversible and energy is dissipated in the solid. The dissipation of the mechanical energy can manifest itself as heat, as permanent deformation or as internal friction, or viscosity, which tends to resist the deformation. Many materials have viscoelastic properties that are both non-linear and time dependent. In particular, composite materials have many distinctive characteristics that cannot be described by linear elastic or viscoelastic theories. The stress-strain and shear stress-strain curves typical of fibre-reinforced composites are manifestly non-linear. The viscoealstic behaviour of composite materials are usually considered to be due to the resin matrix. Hence, any complete theory of the viscoelasticity character of composite materials should be able to describe the local deformation gradient in terms of the forces acting and the material properties. The multidimensional convolution representation provides a natural way to characterise the viscoelastic response of the composite to the applied forces.

The main difficulty in characterising viscoelastic behaviour, from an experimental point of view, is the non-linear and time dependent nature of the data to be analysed. A pre-requisite for any analysis performed is that the characterisation obtained from the data analysis should be related to an appropriate theory underpinning the process. Generally speaking, the ability of current data analysis methods to accurately and consistently quantify the observed behaviour under general dynamic boundary conditions is severely limited. Thus, there is a need to develop and refine data analysis techniques that can separate and quantify the viscoelastic processes and their interactions, and relate them to an appropriate theoretical description of the process.

The notion of inverse equations has been used frequently in the description of the viscoelastic process, usually in the continuous form. In this work a discrete vector multidimensional convolution form of the Volterra series expansion, suitably truncated, is used. A tractable moment hierarchy with well behaved coefficients is obtained by statistical averaging, and the moment hierarchy is solved for the kernel values which characterise the observed viscoelastic behaviour. These kernel functions are usually known as the linear and non-linear response functions and, in the case of a closed physical system, are fundamental properties of the physical system being studied. The Volterra functional expansion is an extension of the Taylor's expansion to those processes which possess a memory. A multidimensional convolution form of the Volterra series is used in the present paper to analyse experimental data for the mixed linear and non-linear properties of viscoelastic behaviour in fibre reinforced composite materials. 
Several viscoelastic theories have used the Volterra functionals to represent the relationship between the components of the stress field $\left\{\sigma_{\mathrm{ij}}(\mathrm{t})\right\}$ and the strain field $\left\{\varepsilon_{\mathrm{ij}}(\mathrm{t})\right\}$; where the component of stress, $\sigma_{i j}(t)$, is considered to be the $i$ th component of the force per unit area on the surface acting in a direction $e_{j}$ which is a unit normal and where $\varepsilon_{i j}(t)$ are the components of the local deformation gradient, $\{\nabla x(t)\}$, the components being $\frac{\partial x_{i}(t)}{\partial X_{j}(t)}$.

The most comprehensive of these theories is by Green and Rivilin [1], who used a tensor form of the Volterra functional series to develop a three dimensional non-linear viscoelastic theory. Their theory is based on the assumption that the observed stress field $\left\{\sigma_{i j}(t)\right\}$, is a non-linear function of the history of observed deformation gradient $\{\nabla x(t)\}$. The values of the stress components $\left\{\sigma_{i j}(t)\right\}$ can be expressed to any desired degree of approximation as an ascending series of convolution functions with

$\sigma_{i j}(t)=\sum_{n=1}^{N} \frac{1}{n !} \int_{0}^{t} d \tau_{1} \ldots \int_{0}^{t} d \tau_{n} E_{i j p 11_{1}-p_{n} q_{n}}\left(\tau_{1}, \ldots, \tau_{n}\right) \prod_{k=1}^{n} g_{p_{k} q_{k}}\left(t-\tau_{k}\right)$

where $\tau_{\mathbf{k}}$ denotes delay with respect to the present time $t$, and where $\mathrm{g}_{\mathrm{p}_{\mathrm{k}} \mathrm{q}_{\mathrm{k}}}\left(\mathrm{t}-\tau_{\mathrm{k}}\right)=\sum_{\mathrm{m}=1}^{9} \frac{\partial \mathrm{x}_{\mathrm{m}}\left(\tau_{\mathrm{k}}\right)}{\partial \mathrm{X}_{\mathrm{p}_{\mathrm{k}}}\left(\tau_{\mathrm{k}}\right)} \frac{\partial \mathrm{x}_{\mathrm{m}}\left(\tau_{\mathrm{k}}\right)}{\partial \mathrm{X}_{\mathrm{q}_{\mathrm{k}}}\left(\tau_{\mathrm{k}}\right)}$ is the inner product of the deformation vector with itself, at the time $\left(t-\tau_{k}\right)$.

The kernels of the convolution expansion represent the dynamic response functions between the stress and strain fields, and they are called the dynamic relaxation modulus functions. The area under these response functions is the steady state gain between the components [2], and are called the relaxation modulus for each pair of components.

The Volterra series representation is well conditioned for a wide range of loading functions. However, Gradowczyk [3] indicated that the Volterra series is ill conditioned under the step loading case. This result is not surprising, as it has long been recognised that the classical test loadings of the step, the single impulse and the single harmonic driving force are not appropriate for non-linear processes [4] and render, for example, the Volterra series and its transformations ill conditioned. Indeed, it was this fact that prompted Weiner [4] to propose that Gaussian white noise could be an appropriate loading function for non-linear systems. 
Unfortunately, it can be trivially shown that a Gaussian white noise loading function produces an ill conditioned Volterra series when the system is of a mixed order higher than quadratic. This is perhaps why the Weiner school developed the homogeneous approximation method which uses Gaussian white noise [5], as that method only used the diagonal terms in Gradowczyk's matrix expression. The objective of the homogeneous approximation is to obtain a local single order radial basis transformation type of description which adequately describes the observed behaviour.

Pipkin and Rogers [6] studied a three dimensional Stieltjes form of the Volterra series expansion and represented the components of the stress field, $\left\{\sigma_{\mathrm{ij}}(\mathrm{t})\right\}$, as a functional expansion with

$\sigma_{i j}(t)=\sum_{n=1}^{N} \frac{1}{n !} \int_{0}^{t} d \varepsilon\left(\tau_{1}\right) \ldots \int_{0}^{t} d \varepsilon\left(\tau_{n}\right) R_{{ }_{i j} p_{1} q_{1} \ldots p_{n} q_{n}}\left(\varepsilon\left(\tau_{1}\right), \ldots, \varepsilon\left(\tau_{n}\right)\right)$

where the differentials

$\mathrm{d} \varepsilon\left(\tau_{1}\right) \ldots \mathrm{d} \varepsilon\left(\tau_{\mathrm{n}}\right) \mathbf{R}_{\mathrm{ij} \mathrm{p}_{1} \mathrm{q}_{1} \cdots \mathrm{P}_{\mathrm{n}} \mathrm{q}_{\mathrm{n}}}\left(\varepsilon\left(\tau_{1}\right), \ldots, \varepsilon\left(\tau_{\mathrm{n}}\right)\right)=\frac{\partial{ }^{\mathrm{n}} \mathrm{R}_{{ }_{\mathrm{ij}} \mathrm{p}_{1} \mathrm{q}_{1}-\mathrm{p}_{\mathrm{n}} \mathrm{q}_{\mathrm{n}}}\left(\varepsilon\left(\tau_{1}\right), \ldots, \varepsilon\left(\tau_{\mathrm{n}}\right)\right)}{\partial \varepsilon\left(\tau_{1}\right) \ldots \partial \varepsilon\left(\tau_{\mathrm{n}}\right)} \prod_{\mathrm{k}=1}^{\mathrm{n}} \frac{\partial \varepsilon\left(\tau_{\mathrm{k}}\right)}{\partial \tau_{\mathrm{k}}} \mathrm{d} \tau_{\mathrm{k}}$

have the properties of a characteristic function [7].

Pipkin and Rogers undertook a detailed examination of the one dimensional case of equation (2) under an incremental step loading scheme. Although reasonable results were obtained, it was later noticed that equation (2) is ill conditioned under the loading regime used, in the same way that the Green and Rivilin expansion is when a step loading is used.

If local solutions are required then the Volterra series can be truncated to just the first (linear) term. The first term approximation has been used by a series of workers, notably Schapery [8], to develop an approximate constitutive theory for composite materials and then to apply their method to a range of material types with generally reasonable agreement between theory and experiment being observed. 
In the Schapery approach, the components of the stress field are related to the temporal differentials of the time series history of deformation gradients. Experimental observations are, by nature, uncertain and their time series are stochastic processes. Most stochastic processes do not posses differentials in the ordinary sense [9], however, a few stochastic processes have differential properties in the mean square, or higher order moment, sense. This indicates that the fundamental relationships between any observed physical quantities should be developed in terms of their time series averaged or convoluted values, and not in terms of the derivatives of the time series values.

More recent work in non-linear elastic dynamics has concentrated on functional analysis and the solution of non-linear differential equations $[10,11]$. The bifurcation and chaotic theories used to describe the non-linear elastic behaviour do not take into account the fading memory properties [12] of viscoelastic processes and consequently will not be considered further in the present work.

The multidimensional convolution representation developed in the present work relates the applied mechanical force to components of the observed deformation gradient, $\left\{\varepsilon_{\mathrm{ij}}(\mathrm{t})\right\}$. The formalism is presented in general terms without specific properties being attributed to the functionals and their coefficients, the response function values. The linear-non-linear response functions of the formalism are estimated directly from the experimental data $[13,2,12]$. The formalism is then applied to experimental data to analyse the viscoelastic process in resin matrix composites under various loading conditions. 


\section{Linear elastic materials}

Before details of the multidimensional convolution formalism are given, it is of value to outline the underlying methodology with a simple example. The theory of linear perfectly elasticity materials is the cornerstone of the macroscopic treatment of solid mechanics. Such ideal materials deform instantaneously in response to an applied load and have the ability to store energy without dissipation, so that all of its stored energy can be recovered. For these materials Hook's law applies, so that the observed stress field if directly proportional to the applied strain and the behaviour is linear. On the other hand, a perfectly viscous fluid has the ability to dissipate energy but not to store it and the stress depends on the rate of change of the strain field.

Real materials have the capacity to both store and dissipate energy and the response to an applied force will be a fast deformation followed by a slow flow process. In a linear viscoelastic material the strain is directly proportional to the strain field and for a given constant applied stress the strain increases with time. This process is known as creep and when the applied force is reduced, or stopped, there is a period of creep recovery when the material experiences strain decay. This is known as relaxation. The phenomena of relaxation and creep are basic characteristics of viscoelastic materials. Any theory that successfully describes the behaviour of viscoelastic materials should be able to characterise the constitutive relationship between the observed deformation and the forces acting and the fluxes flowing. In addition, the theory should be able to characterise the storage and dissipative processes that simultaneously act in the material. The present work attempts to develop such a theory and to illustrate how the coefficients which characterise these relationships can be estimated from experimental data.

As an example of the basis of the methodology underlying the treatment of complex materials, consider a one dimensional linear elastic material that is submitted to a history of mechanical forces in the absence of other forces and thermodynamic fluxes. Then the local applied force, $\{\sigma(t)\}$, can be expressed as a convolution between the local stress, $\{\varepsilon(t)\}$, and the response function, $\mathrm{J}_{\mathrm{E \sigma}}\left(\tau_{1}\right)$, which is called the relaxation modulus. 
For a discrete process which possesses a local fading memory of duration $\mu$, the convolution can be expressed as

$\sigma(t)=\sum_{\tau_{1}=0}^{\mu} J_{\varepsilon \sigma}\left(\tau_{1}\right) \varepsilon\left(t-\tau_{1}\right)$

where $\tau_{1}$ denotes delay with respect to the time $t$. As it stands, it is ill posed because there are $(\mu+1)$ unknowns and only one equation. Hence a set of $(\mu+1)$ equations need to be formed and solved for the response function values, $J_{\varepsilon \sigma}\left(\tau_{1}\right)$. If the local stress, $\{\varepsilon(t)\}$, and the applied force, $\{\sigma(t)\}$, are drawn from stochastic processes, then equation (5) can be operated on to yield the moment equation

$$
\left\langle\varepsilon\left(\mathrm{t}-\varsigma_{1}\right) \sigma(\mathrm{t})\right\rangle=\sum_{\tau_{1}=0}^{\mu} \mathrm{J}_{\varepsilon \sigma}\left(\tau_{1}\right)\left\langle\varepsilon\left(\mathrm{t}-\varsigma_{1}\right) \varepsilon\left(\mathrm{t}-\tau_{1}\right)\right\rangle
$$

where $\left\langle\varepsilon\left(\mathrm{t}-\varsigma_{1}\right) \sigma(\mathrm{t})\right\rangle$ and $\left\langle\varepsilon\left(\mathrm{t}-\varsigma_{1}\right) \varepsilon\left(\mathrm{t}-\tau_{1}\right)\right\rangle$ are the cross and auto moments between the strain field $\{\varepsilon(t)\}$ and the stress field $\{\sigma(t)\}$. That is, the average product of each side with the delayed value of stress, $\varepsilon\left(t-\varsigma_{1}\right)$, has been obtained using the operator $\left\langle\varepsilon\left(t-\varsigma_{1}\right) *\right\rangle$ for $0 \leq \tau_{1} \leq \mu$ to give the $(\mu+1)$ equations required. In this form the equations, given by (6), can be readily solved with standard matrix methods.

Under steady state conditions the one dimensional linear stress-strain relationship becomes

$$
\sigma=\varepsilon \sum_{\tau_{1}=0}^{\mu} \mathrm{J}_{\varepsilon \sigma}\left(\tau_{1}\right)
$$

that is $\sigma=\varepsilon \mathrm{J}_{\varepsilon \sigma}^{*}$, where $\mathrm{J}_{\varepsilon \sigma}^{*}$ is the steady relaxation modulus. If now the load is incremented form the steady state value by an amount $\Delta \sigma$ then after the stress field will be

$$
\sigma^{*}=\varepsilon J_{\varepsilon \sigma}^{*}+\sum_{\tau_{1}=0}^{\mu} J_{\varepsilon \sigma}\left(\tau_{1}\right) \Delta \varepsilon\left(t-\tau_{1}\right)
$$

After $\mu$ units of time have elapsed, the strain field will be given by

$$
\sigma^{*}=\varepsilon J_{\varepsilon \sigma}^{*}+\Delta \varepsilon J_{\varepsilon \sigma}^{*}
$$

which satisfies the Boltzman superposition principle for a linear viscoelastic process. 
Thus, the general linear stress strain expression for an arbitrary sequence of loading forces is the convolution equation

$$
\sigma(t)=\sum_{\tau_{1}=0}^{\mu} J_{\varepsilon \sigma}\left(\tau_{1}\right) \varepsilon\left(t-\tau_{1}\right)
$$

However, it should be noted that this representation does not satisfy the causality relationship that exists in the physical situation.

Alternatively the stress can be characterised as a linear function of the applied force

$$
\varepsilon(t)=\sum_{\tau_{1}=0}^{\mu} C_{\sigma \varepsilon}\left(\tau_{1}\right) \sigma\left(t-\tau_{1}\right)
$$

Under steady state conditions this becomes

$$
\varepsilon=\sigma \sum_{\tau_{1}=0}^{\mu} \mathrm{C}_{\sigma \varepsilon}\left(\tau_{1}\right)=\sigma \mathrm{C}_{\sigma \varepsilon}^{*}
$$

\section{Volterra functional series representation of viscoelasticity}

There are many physical processes where the form of the differential equations that govern the observed behaviour are not known. In such cases other representations must be used to describe the physical process. For example, the fields of thermodynamics, fluid dynamics and elasticity use truncated Taylor's series expansion representations. When the Taylor's series expansion description is used, the physical laws that describe aspects of the observed behaviour can be based on the values of the coefficients of the ascending order terms in the expansion.

Constitutive equations are expressions which characterise the observed behaviour between forces and fluxes and conservation expressions relate a conserved variable to the constituent variables. For example, an observed thermodynamic flux may be characterised in terms of the observed thermodynamic forces and observed properties of the medium. The empirical coefficients of the Taylor's series expansion describe the steady state transport properties of the process. Such empirical coefficients represent the, so called, steady state gains of the independent variable to the dependent variables and cannot be derived from any fundamental theory, but are estimated directly from the experimental data. 
The constitutive equations which describe thermodynamic processes, each thermodynamic flux, $f_{k}(t)$, can be described as a multidimensional convolution expansion in terms of the local thermodynamic forces acting, and defined as

$$
f_{k}(t)=\sum_{n=1}^{N} \frac{1}{n !} \sum_{i_{1}=1}^{I} \ldots \sum_{i_{n}=i_{n-1}}^{I} \int_{t-\mu}^{t} d \tau_{1} \ldots \int_{t-\mu}^{t} d \tau_{n} J_{f_{k} F_{i_{1}}-F_{i_{n}}}\left(\tau_{1}, \ldots, \tau_{n}\right) \prod_{j=1}^{n} F_{i_{j}}\left(t-\tau_{j}\right)
$$

where $\mathrm{N}$ is the order of the system, where t denotes time and where the $\tau_{\mathrm{j}}$ 's denotes time delay with respect to the time $t$.

In this work we consider the univariate form which is used to relate the applied force to the stress field, explicitly

$$
\sigma(t)=\sum_{n=1}^{N} \frac{1}{n !} \int_{t-\mu}^{t} d \sigma_{1} \ldots \int_{t-\mu}^{t} d \sigma_{n} J_{\sigma \varepsilon^{n}}\left(\tau_{1}, \ldots, \tau_{n}\right) \prod_{j=1}^{n} \varepsilon\left(t-\tau_{j}\right)
$$

is considered.

A discrete approximation to the multidimensional convolution expansion can be defined as

$$
\sigma(t)=\sum_{n=1}^{N} \frac{1}{n !} \sum_{\tau_{1}=0}^{\mu} \ldots \sum_{n}^{\mu} J_{\sigma \varepsilon^{n}}\left(\tau_{1}, \ldots, \tau_{n}\right) \prod_{j=1}^{n} \varepsilon\left(t-\tau_{j}\right)
$$

where $\mathrm{N}$ is the order of truncation the system and where $\mu$ is the finite memory of the process.

On discretisation, the truncated Volterra series remains ill posed in the sense that there are too many unknown coefficients to solve for. Thus, the approximate method of discretisation used for the linear case cannot by itself be used to solve the Volterra series. A tractable set of simultaneous equations with well behaved coefficients can be generated by taking time series moments of a suitably truncated Volterra series expansion.

Integrating each kernel function yields the linear and non-linear gain between the dependent and independent variables [2], with

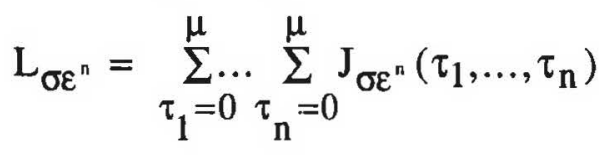


That is, the integral of the kernel function values yields the steady state gain between the observables and are equivalent to the ascending order transport coefficients of the phenomena being characterised.

Equations (12) is ill posed, in the sense that there are many coefficients to solve for with only one equation. In addition, as thermodynamic processes are stochastic, in general, the equation is also ill conditioned because it has stochastic variables.

The conditioning can be improved statistical averaging and the use of operators allows a set of tractable equations with average variable values to be generated. Equation (12) is operated on with a series of averaging operators, one for each permutation of delayed applied forces $\left\langle\prod_{i=1}^{n} \varepsilon\left(t-\varsigma_{i}\right)^{*}\right\rangle$ which yields the moment hierarchy for viscoelasticy

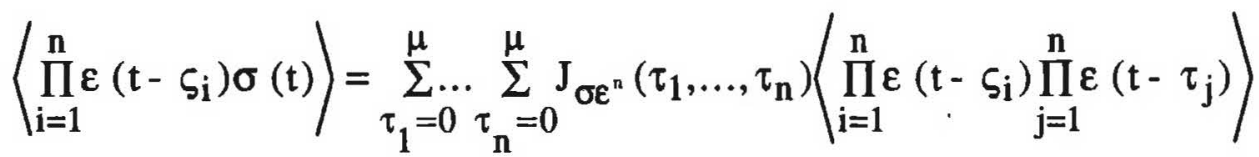

where $\langle *\rangle$ denotes the averaging operation. The moment hierarchy can be rewritten in the obvious matrix form $\underline{\mathrm{C}}=\underline{\underline{\mathrm{M}}} \underline{\underline{h}}$ where $\underline{\underline{M}}$ is a square matrix whose elements are the automoments of the observed stress, $\{\varepsilon(t)\}$, where $\underline{C}$ is a column vector whose elements are the cross moments between the stress, $\{\varepsilon(t)\}$ and the applied force $\{\sigma(t)\}$ and where $\underline{h}$ is a column vector whose elements are the kernel function values of the mapping between $\{\sigma(t)\}$ and $\{\varepsilon(t)\}$.

If the matrix $\underline{\underline{\mathrm{M}}}$ is non-singular then $\underline{\underline{h}}=(\underline{\underline{\mathrm{M}}})^{-1} \underline{\mathrm{C}}$ has a unique solution. If however $\underline{\underline{\mathrm{M}}}$ is singular, then $\underline{\underline{M}}$ is rank deficient and some of its rows will be linearly dependent on the others. If the same relationship holds between the corresponding elements of the column vector $\underline{C}$, the solution will not be unique, indeed an infinity of solutions will exist. If this is not the case then the matrix expression is not consistent and there will not be any solution. Thus, in general, there may be a unique solution, an infinite number of solutions or no solution. However, given the construction of the moment values used in the moment hierarchy, the rows of $\underline{M}$ will be linearly independent of each other, thus the matrix will usually be non-singular and have a unique solution. This will be true for many mixed stochastic and deterministic processes. 
At this stage an observation can be made. The Volterra functional expansion is not, in general, tractable. However, the application of averaging operators has generated a tractable hierarchy of moment equations which are likely to have well behaved coefficients for many physical processes. The truncated Volterra expansion has been operated on in order to obtain a linear algebraic expression where the elements of the vector and matrix retain all of the information about the complex dynamical non-linear process being studied.

There are exceptions to this however, for example,

1) when the data $\{x(t)\}$ are composed of delta function, a step function, and for any distribution which has a delta functional form of each member of the ascending order of auto moments, or their transformation, of the data $\{x(t)\}$

2) when the data $\{x(t)\}$ at successive time series points are not causally linked

3) when the data $\{x(t)\}$ are composed of a very nonstationary sequence and

4) when the representation of the process is not of a closed form, i.e. some of the contributing variables are not measured or analysed. 


\section{Facilities and analysis of the viscoelasticity experiments}

A series of test coupons were manufactured from glass/polyester composite and were representative of typical wind turbine blade design. Two types of GRP coupon were investigated, a $0^{\circ}-90^{\circ}$ with a $90 / 10$ ratio of weave in the fabric and a balanced $45^{\circ}$ fabric. In addition a curved plate section, cut from an existing turbine blade, was studied under uniaxial loading. The curved plate and the 90-10 fibre reinforced specimens were all cut from existing wind turbine blades manufactured by L/M Glassfibre who are collaborators in the present project. Dynamic fatigue loading is normally used to investigate the nature of the failure mechanisms of the material under study. Such accelerated lifetime methods provide fatigue life endurance and fatigue limit, but have the disadvantage that the results can be unrealistic. In this work fatigue loadings are used primarily to obtain time series sequences suitable for mixed linear-non-linear analysis. Notwithstanding the anticipated shortcomings of the fatigue method it is shown that realistic estimates of the material properties can be obtained from the time series analysis. It is well known that such GPR composite materials behave in a non-linear viscoelastic manner and when the material is fatigue loaded energy is dissipated as heat. This heating effect is ignored in the present paper but is considered in detail in an accompanying paper.

In order to compare the findings of the present work with the standard summary statistic (the Young's modulus) it is assumed in the analysis that the applied load is a mixed linearnon-linear dynamical function of the observed stress. The most commonly used excitation function is the sinusoid which is used in the present work. The fatigue loading on the specimens used in the present work was only approximately sinusoidal because of the nonlinear fluid interactions in the hydraulic system which coupled several different experimental rigs in the laboratory. Time series measurements were collected with a personnel computer through a data collection card. For each separate experiment some 4000 time series points for each sensor were collected. Of these some 500, in sample, points were used to estimate the response factor values of the process and some 3000 , out of sample, points were used to compare with the values of the strain field values predicted using the response factor values estimated in sample. This enabled the accuracy and time invariant nature of the estimated response function values to be determined. These response function values were then used to predict the behaviour of the sample under a different loading regime, thus testing the nature of the solutions determined by the moment hierarchy method. 
The time series values of the stress field were considered 1) as a linear function of the applied load and 2) as a mixed linear and non-linear function of the applied load applied to the solid. In each case the properties of the process were characterised with 500 data points. The response functions estimated with these 500 points were then used to predict the future, out of sample, behaviour of the stress field for 3000 points with no obvious sign of dispersion between the predicted and the measured points.

These predicted values of stress, $\left\{\varepsilon_{\mathrm{p}}(\mathrm{t})\right\}$, were then compared statistically with the observed values, $\{\varepsilon(\mathrm{t})\}$. It should be stressed that during the prediction phase no use was made of the observed stress values. It should also be noted that the mean value had to be subtracted from the data in order to perform the linear analysis, no such modification was made in the non-linear analyses. This provides a quantitative measure of the quality of the response function characterisation of the viscoelastic process. The accuracy of the predicting ability was determined by comparing the root mean square differences between the actual, $\{\varepsilon(t)\}$, and predicted $\left\{\varepsilon_{\mathrm{p}}(\mathrm{t})\right\}$, time series sequences. A typical example of the prediction together with the actual values is shown in figure 1 .

The results for the balanced $45^{\circ}$ GRP sample under various uniaxial compressive loadings are presented. Sample estimates of the response function values were obtained in sample. Out of sample rolling predictions were then compared with the observed data. The Student's $\mathrm{t}$-test values determined in that analysis are presented in table 1 below. These out of sample predictions provide a sensitive and accurate measure of the quality of the response function characterisation of the viscoelastic process.

\section{Compressive experiments on uniaxial balanced $45^{\circ}$ GRP sample}

Table 1: Student's t-test value for the predicted and actual stress values

\begin{tabular}{|l|l|l|l|}
\hline $\begin{array}{l}\text { Applied } \\
\text { Force } \\
@ 1 \mathrm{~Hz}\end{array}$ & Linear analysis & $\begin{array}{l}\text { Mixed linear- } \\
\text { quadratic analysis }\end{array}$ & $\begin{array}{l}\text { Mixed linear- } \\
\text { quadratic-cubic } \\
\text { analysis }\end{array}$ \\
\hline $1 \leq \mathrm{f} \leq 10 \mathrm{kN}$ & $39 . * 10^{-3}$ & $6.7 * 10^{-3}$ & $6.7 * 10^{-3}$ \\
\hline $1 \leq \mathrm{f} \leq 14 \mathrm{kN}$ & $38 . * 10^{-3}$ & $4.3 * 10^{-3}$ & $4.3 * 10^{-3}$ \\
\hline $1 \leq \mathrm{f} \leq 20 \mathrm{kN}$ & $40 . * 10^{-3}$ & $4.0 * 10^{-3}$ & $4.0 * 10^{-3}$ \\
\hline
\end{tabular}


The data were analysed for estimates of the response function values of

$\sigma(t)=\sum_{n=1}^{N} \frac{1}{n !} \sum_{\tau_{1}=0}^{\mu} \ldots \sum_{n}^{\mu}=0$

Linear, mixed linear-quadratic and mixed linear-quadratic-cubic analyses yielded the response function values. The Students t-test values given in table 1 show that only the mixed linear-quadratic-cubic non-linear hypothesis is not rejected. Considering only the results from that analysis, integrating each kernel function yields the linear and non-linear gain between the dependent and independent variables and these were used to generate the stress-strain curves shown in figure 2 using

$$
\sigma=\sum_{n=1}^{N} \frac{1}{n !} \prod_{j=1}^{n} \varepsilon^{n}\left\{\sum_{\tau_{1}=0}^{\mu} \ldots \sum_{\tau_{n}=0}^{\mu} J_{\sigma^{n}}\left(\tau_{1}, \ldots, \tau_{n}\right)\right\}
$$

where $\mathrm{N}=3$ is the order of truncation the system and where $\mu$ is the finite memory of the process. Effective stress-strain curves were generated from the response functions estimated in the linear-quadratic-cubic analysis. The values for the Young's modulus determined from these stress-strain curves for the balanced $45^{\circ}$ GRP coupon under uniaxial loadings are given in table 2 below.

Table 2: Effective Young's modulus determined from the stress-strain curves

\begin{tabular}{|l|l|}
\hline $\begin{array}{l}\text { Applied Force } \\
@ 1 \mathrm{~Hz}\end{array}$ & $\begin{array}{l}\text { Linear-quadratic-cubic } \\
\text { non-linear analysis }\end{array}$ \\
\hline $1 \leq \mathrm{f} \leq 20 \mathrm{kN}$ & $15300 \pm 400 \mathrm{~N} \mathrm{~mm}^{-2}$ \\
\hline $1 \leq \mathrm{f} \leq 40 \mathrm{kN}$ & $14360 \pm 400 \mathrm{~N} \mathrm{~mm}^{-2}$ \\
\hline $1 \leq \mathrm{f} \leq 60 \mathrm{kN}$ & $13750 \pm 400 \mathrm{~N} \mathrm{~mm}^{-2}$ \\
\hline static test $\mathrm{E}_{11}$ & $15000 \pm 400 \mathrm{~N} \mathrm{~mm}^{-2}$ \\
\hline
\end{tabular}

These estimated values agree well within the statistical uncertainties but the curves in figure 2 clearly show an excess strain factor as the applied load is increased. The uncertainties quoted arise from the accuracy of the sensors used in the experiments. This excess strain is accompanied with an increasing heat flux observed at the surface of the samples under test. The coupled thermoviscoelastic effect is considered in detail elsewhere. 
Next the results for the $0^{\circ}-90^{\circ} \mathrm{GRP}$ sample under various uniaxial compressive and then compresive-tensile loadings are presented. As before, sample estimates of the response function values were obtained in sample. Out of sample rolling predictions were then compared with the observed data. The Student's t-test values determined in that analysis are presented in table 3 below.

Table 3: Student's t-test value for the $0^{\circ}-90^{\circ} \mathrm{GRP}$ sample

\begin{tabular}{|l|l|l|l|}
\hline $\begin{array}{l}\text { Applied Force } \\
@ 1 \mathrm{~Hz}\end{array}$ & Linear analysis & $\begin{array}{l}\text { Mixed linear- } \\
\text { quadratic analysis }\end{array}$ & $\begin{array}{l}\text { Mixed linear- } \\
\text { quadratic-cubic } \\
\text { analysis }\end{array}$ \\
\hline $1 \leq \mathrm{f} \leq 20 \mathrm{kN}$ & -7.8 & -4.3 & -0.09 \\
\hline $1 \leq \mathrm{f} \leq 40 \mathrm{kN}$ & -7.7 & -4.4 & -2.8 \\
\hline $1 \leq \mathrm{f} \leq 60 \mathrm{kN}$ & -9.4 & -9.1 & 3.0 \\
\hline$-5 \leq \mathrm{f} \leq 25 \mathrm{kN}$ & -0.79 & -1.38 & 0.14 \\
\hline$-5 \leq \mathrm{f} \leq 45 \mathrm{kN}$ & -11.5 & -13.0 & 0.64 \\
\hline$-5 \leq \mathrm{f} \leq 65 \mathrm{kN}$ & -11.2 & -13.0 & 2.7 \\
\hline
\end{tabular}

Linear, mixed linear-quadratic and mixed linear-quadratic-cubic analyses using equation (18) yielded the response function values, from which the stress-strain curves shown in figures 3 and 4 were calculated. The Student's t-test values given in tables 3 and 4 show that, using the union-intersection principle of statistics, only the mixed linear-quadratic-cubic non-linear hypothesis is not rejected. Effective stress-strain curves were generated from the response functions estimated in the linear-quadratic-cubic analysis. The values for the Young's modulus determined from these stress-strain curves the $0^{\circ}-90^{\circ} \mathrm{GRP}$ sample under various uniaxial compressive and then compresive-tensile loadings are given in table 4 below. 
Table 4: Effective Young's modulus determined from the stress-strain curves

\begin{tabular}{|l|l|}
\hline $\begin{array}{l}\text { Applied Force } \\
@ 1 \mathrm{~Hz}\end{array}$ & $\begin{array}{l}\text { Linear-quadratic-cubic } \\
\text { non-linear analysis }\end{array}$ \\
\hline $1 \leq \mathrm{f} \leq 20 \mathrm{kN}$ & $31600 \pm 800 \mathrm{~N} \mathrm{~mm}^{-2}$ \\
\hline $1 \leq \mathrm{f} \leq 40 \mathrm{kN}$ & $31300 \pm 800 \mathrm{~N} \mathrm{~mm}^{-2}$ \\
\hline $1 \leq \mathrm{f} \leq 60 \mathrm{kN}$ & $31200 \pm 800 \mathrm{~N} \mathrm{~mm}^{-2}$ \\
\hline$-5 \leq \mathrm{f} \leq 25 \mathrm{kN}$ & $28800 \pm 800 \mathrm{~N} \mathrm{~mm}^{-2}$ \\
\hline$-5 \leq \mathrm{f} \leq 45 \mathrm{kN}$ & $29000 \pm 800 \mathrm{~N} \mathrm{~mm}^{-2}$ \\
\hline$-5 \leq \mathrm{f} \leq 65 \mathrm{kN}$ & $29300 \pm 800 \mathrm{~N} \mathrm{~mm}^{-2}$ \\
\hline static test $\mathrm{E}_{11}$ & $24000 \pm 2000 \mathrm{~N} \mathrm{~mm}^{-2}$ \\
\hline
\end{tabular}

The Young's modulus values obtained from the fatigue testing are self consistent but systematically higher in magnitude than those determined in the static test. However, during the static testing of several 90-10 coupons the stress strain curves were irregular and nonlinear, consequently there was a wide spread in the values obtained. 
Finally the results for the curved plate under various uniaxial compressive-tensile loadings are presented. Repeat experiments were performed at the same fatigue loading to investigate the consistency of the analysis method being used. As before, sample estimates of the response function values were obtained in sample. Out of sample rolling predictions were then compared with the observed data. The Student's $t$-test values determined in that analysis are presented in table 5 below.

Table 5: Student's t-test value for the curved plate compressive-tensile loadings

\begin{tabular}{|l|l|l|l|}
\hline $\begin{array}{l}\text { Applied Force } \\
@ 1 \mathrm{~Hz}\end{array}$ & Linear analysis & $\begin{array}{l}\text { Mixed linear- } \\
\text { quadratic analysis }\end{array}$ & $\begin{array}{l}\text { Mixed linear- } \\
\text { quadratic-cubic } \\
\text { analysis }\end{array}$ \\
\hline$-15 \leq \mathrm{f} \leq 5 \mathrm{kN}$ & 51.0 & -9.34 & -3.28 \\
\hline$-15 \leq \mathrm{f} \leq 5 \mathrm{kN}$ & 44.2 & -1.67 & 5.72 \\
\hline$-15 \leq \mathrm{f} \leq 5 \mathrm{kN}$ & 48.8 & 2.02 & 0.94 \\
\hline$-15 \leq \mathrm{f} \leq 5 \mathrm{kN}$ & 50.5 & -12.9 & -5.91 \\
\hline$-15 \leq \mathrm{f} \leq 5 \mathrm{kN}$ & 42.7 & 1.14 & 4.77 \\
\hline$-15 \leq \mathrm{f} \leq 5 \mathrm{kN}$ & 51.0 & -1.44 & 1.16 \\
\hline
\end{tabular}

Linear, mixed linear-quadratic and mixed linear-quadratic-cubic analyses yielded the response function values. Again, effective stress-strain curves were generated from the response functions estimated in the linear-quadratic-cubic analysis. These values are given in table 6 below. 
Table 6: Effective Young's modulus determined from the stress-strain curves

\begin{tabular}{|l|l|}
\hline $\begin{array}{l}\text { Applied Force } \\
@ 1 \mathrm{~Hz}\end{array}$ & $\begin{array}{l}\text { Linear-quadratic-cubic } \\
\text { non-linear analysis }\end{array}$ \\
\hline$-15 \leq \mathrm{f} \leq 5 \mathrm{kN}$ & $29800 \pm 800 \mathrm{~N} \mathrm{~mm}^{-2}$ \\
\hline$-15 \leq \mathrm{f} \leq 5 \mathrm{kN}$ & $27380 \pm 800 \mathrm{~N} \mathrm{~mm}^{-2}$ \\
\hline$-15 \leq \mathrm{f} \leq 5 \mathrm{kN}$ & $26700 \pm 800 \mathrm{~N} \mathrm{~mm}^{-2}$ \\
\hline$-15 \leq \mathrm{f} \leq 5 \mathrm{kN}$ & $30200 \pm 800 \mathrm{~N} \mathrm{~mm}^{-2}$ \\
\hline$-15 \leq \mathrm{f} \leq 5 \mathrm{kN}$ & $27740 \pm 800 \mathrm{~N} \mathrm{~mm}^{-2}$ \\
\hline$-15 \leq \mathrm{f} \leq 5 \mathrm{kN}$ & $27660 \pm 800 \mathrm{~N} \mathrm{~mm}^{-2}$ \\
\hline
\end{tabular}

The Young's modulus values agree within the experimental uncertainties and are self consistent. In addition they lie close to the manufacturer's quoted value for the Young's modulus of their typical glass fibre reinforced resins for which $E_{11}=22270 \mathrm{~N} \mathrm{~mm}^{-2}$ for the curved plate sample used in the present work.

In summary, the linear and mixed linear-quadratic non-linear analyses of the differences between the measured, $\{\varepsilon(t)\}$, and predicted, $\left\{\varepsilon_{\mathrm{p}}(\mathrm{t})\right\}$, output stress field for both modelled and predicted data lay in the rejection region on the basis of a two tailed 5\% probability level of rejection. However, the values of the test statistics for the differences between the measured, $\{\varepsilon(t)\}$, and predicted, $\left\{\varepsilon_{p}(t)\right\}$, output stress field for both modelled and predicted data lay within the acceptance region; thus each representation accurately characterises the observed behaviour of the stress. That is, the sample statistics for the mixed-linear-quadratic-cubic non-linear analyses were not rejected at the two tailed $5 \%$ level. In addition the value of the test statistic can be used as strength of evidence for acceptance of the viscoelastic representation used here. 


\section{Conclusions}

The results presented in this paper can be summarised as follows: a hierarchy of moment equations of the Volterra series can used to study non-linear viscoelastic process in complex materials. The nature of one dimensional viscoelasticity was considered. Linear and mixed linear-quadratic-cubic non-linear local constitutive representations were used to characterise the viscoelastic process of a composite solid under a range of applied loads. The first, second and third order response functions were estimated from the time series data collected from the applied force and strain gauge values. These estimated response functions were then used to predict the out of sample stress field values. These predictions demonstrated that the response functions provided a good non-linear representation of the viscoelastic process.

The analysis has demonstrated that the moment hierarchy can extract and isolate linear and ascending order non-linear response functions when the input data are drawn from a stochastic process. The results of the linear and mixed linear-non-linear analyses of the one dimensional viscoelastic data show that the process is, within the experimental uncertainties, non-linear. The results also indicate that the effect of the thermal forces acting, which also arise from the applied fatigue load, should be considered in the determination of the material properties. The combined thermoviscoelastic properties are considered in detail in an accompanying paper.

\section{Acknowledgements}

The authors would like to acknowledge that this work was funded by the CEC Non Nuclear Energy Program JOULE II. We are also pleased to acknowledge the assistance and helpful comments from our collaborators B R Clayton, D van Delft, A G Dutton, G M Smith and A Yaghi. 


\section{References.}

[1] Green A E and Rivlin R S, The Mechanics of Non-linear Materials with Memory, Arch. Ration. Mech. and Anal., Vol. 1 (1), 1957, p 1-21.

[2] Irving A D, Stochastic Sensitivity Analysis, Applied Mathematical Modelling, Vol. 16, January, 1992, p 3-15.

Gradowczyk M H, On the Accuracy of the Green-Rivlin Representation for Viscoelastic Materials, Int. J. Solids and Struct., Vol. 5 (8), 1969, p 873-877.

[4] Wiener N. Nonlinear problems in random theory. John Wiley, 1958.

[5] Schetzen M, The Wiener and Volterra Theories of Nonlinear Systems, John Wiley, New York, 1980.

[6] Pipkin A C and Rogers T G, A Non-linear Integral Representation for Viscoelastic Behaviour, J. of the Mechanics and Physics of Solids, Vol. 16, 1968 , p 59-74.

[7] Lukas E. and Laka R. G., Applications of characteristic functions, Charles Griffin and Co. Ltd., London, 1964.

[8] Lou YC and Schapery RA, Viscoelastic characterisation of nonlinear fibre reinforced plastic, J. Composite Materials, Vol 5, 1971, p 208-234

[9] Yaglom, An introduction to the theory of stationary random functions, Prentice Hall, Englewood Cliffs, 1962

[10] Marsden JE and Hughes TJR, Mathematical foundations of elasticity, Prentice Hall, Englewood Cliffes, New Jersey, 1983

[11] Ottino JM, The kenematics of mixing: stretching, chaos and transport, Cambridge University Press, Cambridge, 1989

[12] Irving A D and Dewson T, On the analysis of complex time series, Submitted to App. Math. Modelling., 1994.

[13] Irving A D, Dewson T, Hong G and Cunliffe N, General nonlinear response of a single input system to stochastic excitations, In Press App. Math. Modelling., 1994. 


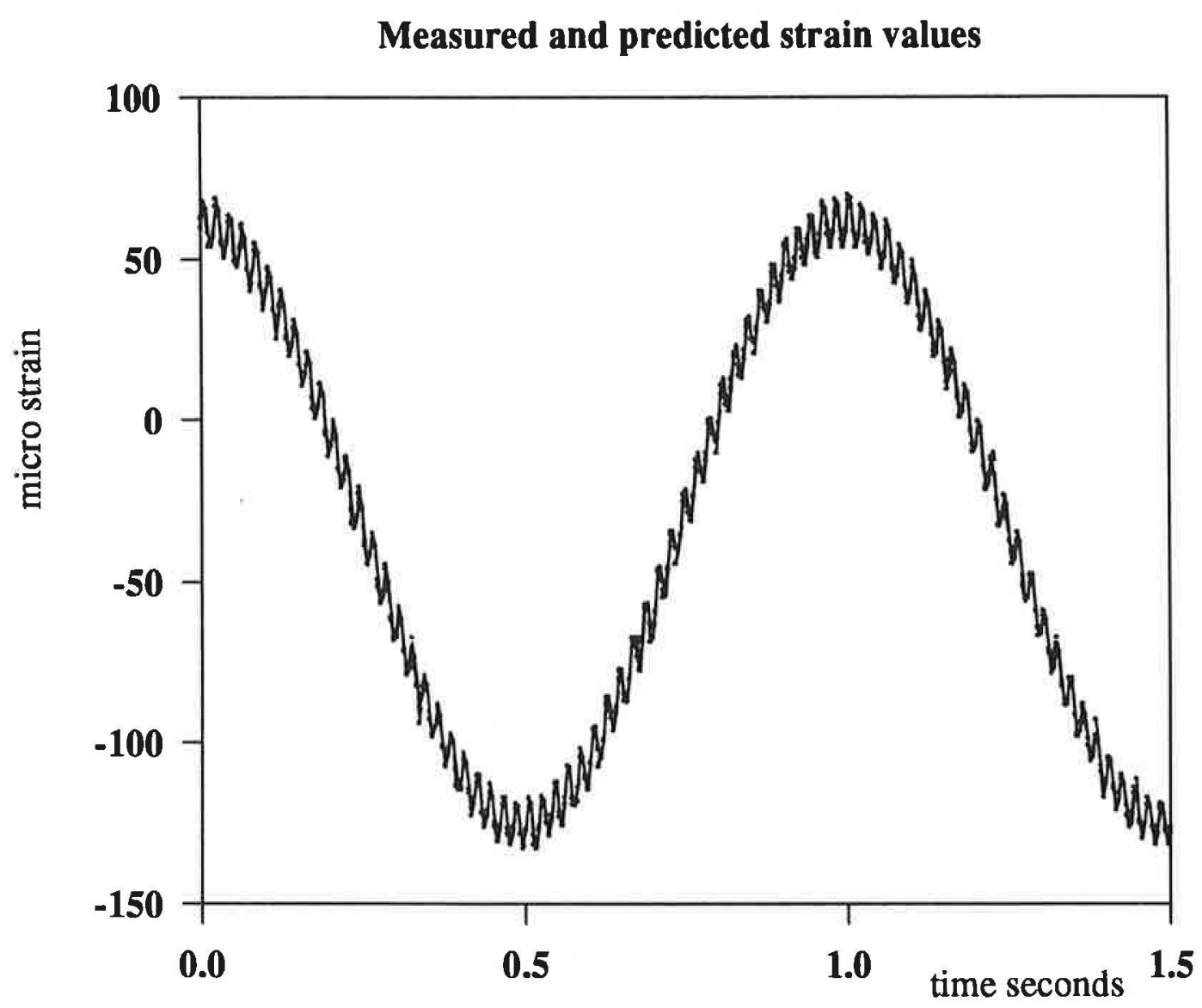

(Measured - Predicted) difference values

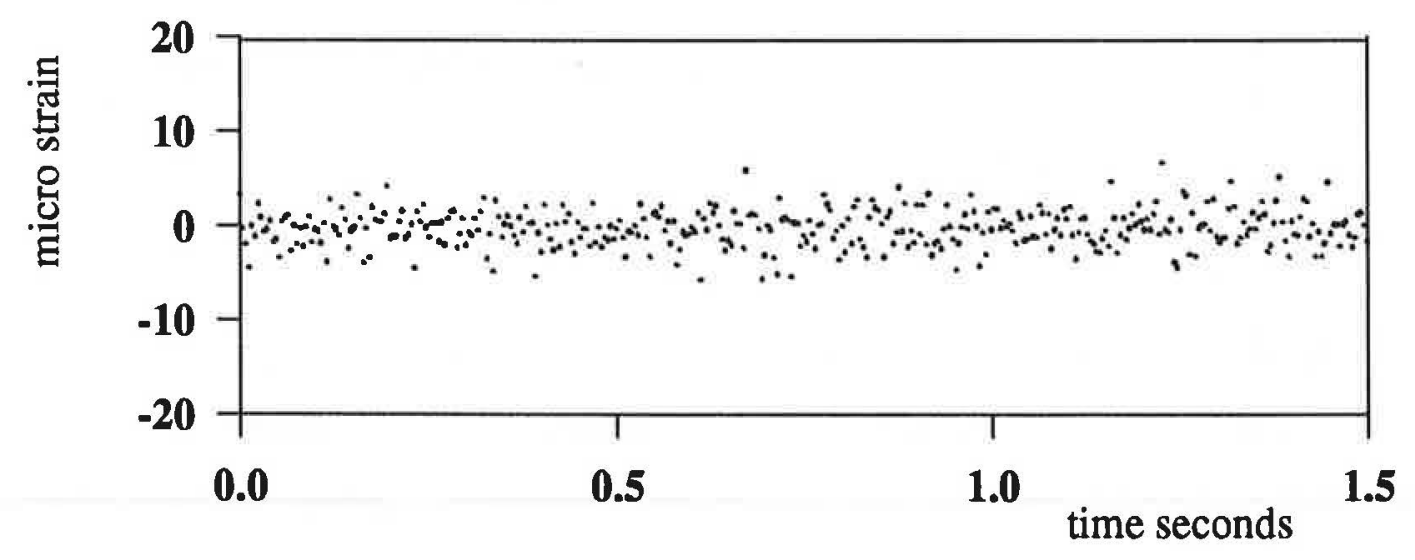




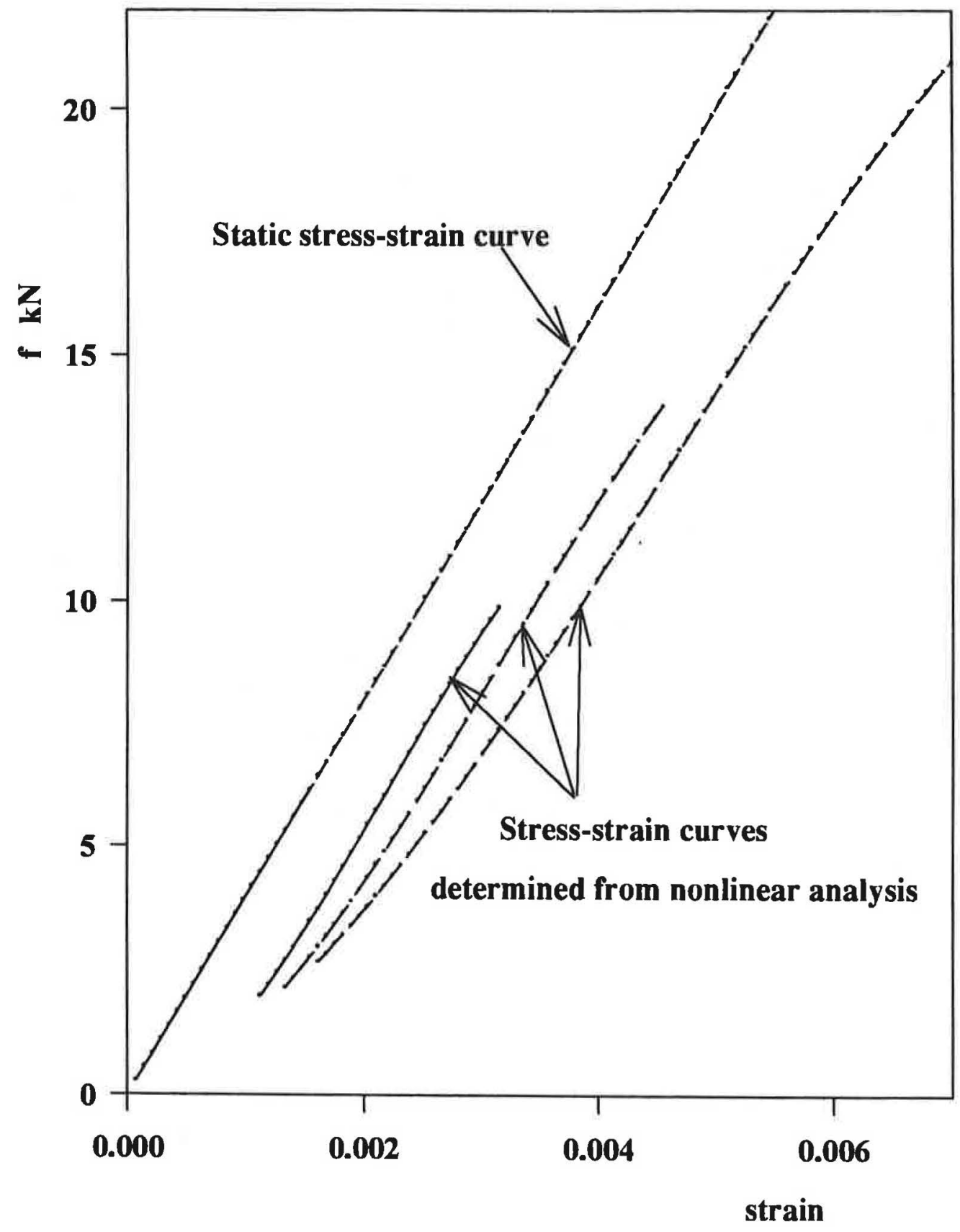




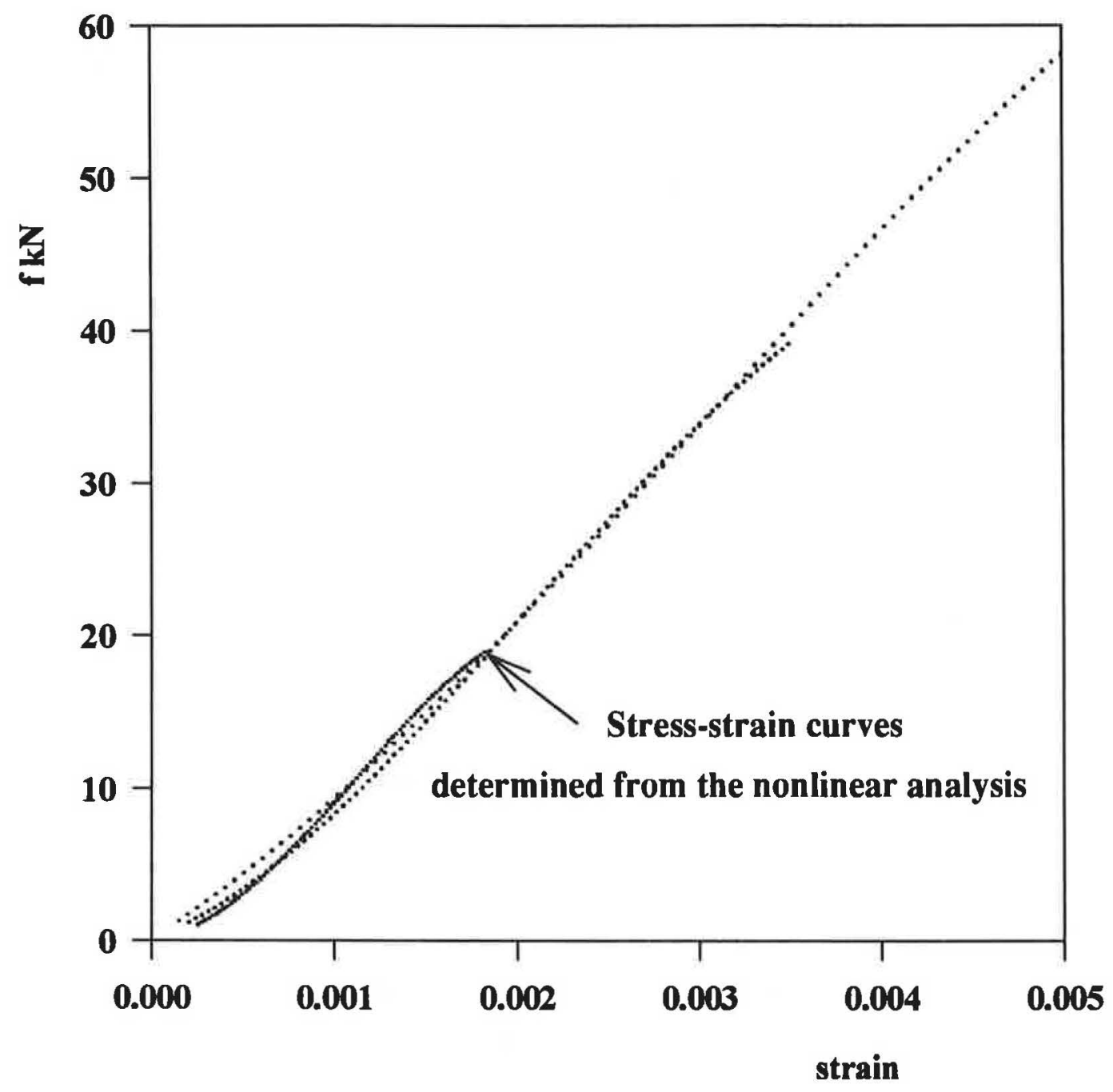




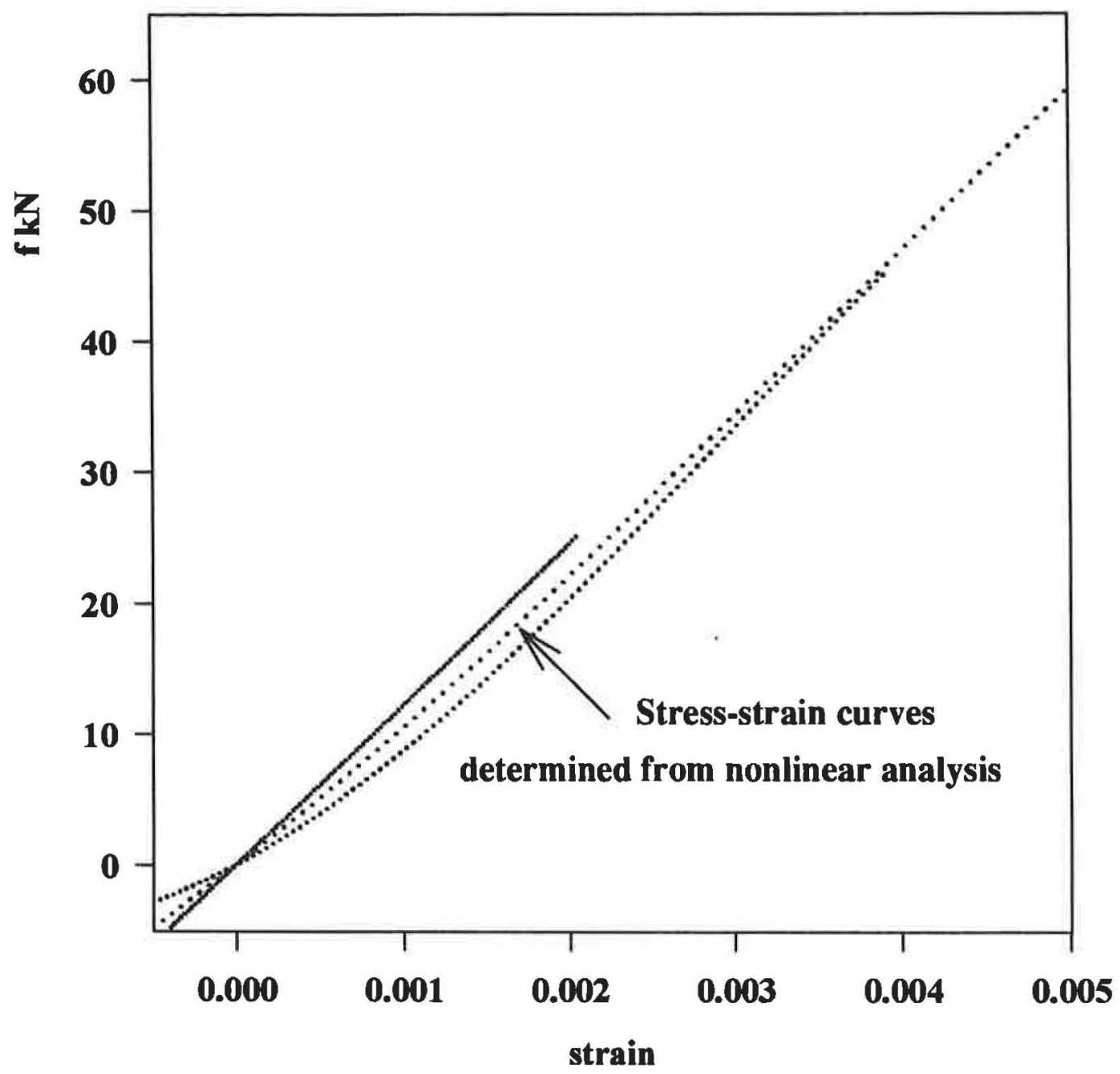



